\title{
Recurrent Unilateral Pleural Effusion from Constrictive Pericarditis of Unknown Etiology Requiring Pericardiectomy
}

\author{
Aishah Ali, MD, Gina Keiffer, MD
}

\section{INTRODUCTION}

Constrictive pericarditis is an uncommon cause of unilateral pleural effusion. In patient's who have repeated thoracenteses with no obvious cause for the pleural effusion, constrictive pericarditis should be considered. Right and left heart catheterization is used to diagnosis constrictive pericarditis by measuring filling pressures of the heart.

\section{CASE REPORT}

A 52-year-old man with a history of hepatitis C, hepatocellular carcinoma $(\mathrm{HCC})$, status post liver transplant in July 2013, chronic kidney disease, gastroesophageal reflux disease and hypothyroidism presented with increasing dyspnea with minimal exertion and was found to have recurrent pleural effusion. Patient had been worked up as an outpatient for recurrent pleural effusion but no etiology had been found. Prior thoracentesis on three different occasions within a month had yielded exudative fluid with no evidence of malignant cells. The effusions re-accumulated within one week on each occasion. The patient had previously been treated with diuretics without resolution of his recurrent pleural effusion. With worsening of his renal function, diuretics had recently been discontinued. The patient denied shortness of breath at rest, cough and chest pain as well as fevers and chills. He also denied orthopnea and paroxysmal nocturnal dyspnea. Medications included tacrolimus, levothyroxine, omeprazole and a daily multivitamin. The patient has a history of prior alcohol abuse and prior tobacco use (10 pack years).

The patient's vital signs were significant for mild tachypnea (20 respirations per minute) with normal oxygen saturation. He initially appeared healthy and in no acute distress. He had jugular venous distention. Pulmonary exam was clear on the left with decreased breath sounds in the right mid- and lower-lung fields. There was mild, bilateral lower extremity pitting edema.

The patient's renal function was at his baseline (creatinine $=1.8 \mathrm{mg} / \mathrm{dL}$, normal range 0.7 - 1.4). Complete blood count identified leukopenia, mild normocytic anemia, and thrombocytopenia. The patient's labs identified elevated pro-brain natriuretic peptide $(2511 \mathrm{pg} / \mathrm{mL}$, normal range $<125 \mathrm{pg} / \mathrm{ml}$ ) and normal hepatic function panel except mildly elevated total bilirubin $(1.3 \mathrm{mg} /$ $\mathrm{dL}$, normal range $0.1-0.9 \mathrm{mg} / \mathrm{dl}$ ). Chest $X$-ray in the Emergency Department identified a large right pleural effusion, increased from a study one week prior and associated right basilar atelectasis as well as a small left pleural effusion and background pulmonary edema. The patient was admitted and work-up for recurrent unilateral pleural effusion was initiated.

\section{DIFFERENTIAL DIAGNOSIS}

Differential diagnosis for an exudative unilateral effusion includes infectious etiologies, including tuberculosis and parapneumonic effusion. Additionally, there was concern for a malignant effusion secondary to the patient's history of HCC.

\section{HOSPITAL COURSE}

The patient's shortness of breath worsened over the first few days of hospital stay. He became more volume overloaded, with $2+$ pitting edema in lower extremities and increasing ascites. He had a therapeutic thoracentesis every other day for three total occasions with a liter of pleural fluid removed each time. Pleural fluid labs showed an alkaline $\mathrm{pH}$ (7.63, normal 7.6 - 7.64), slightly elevated glucose (114 mg/dL, normal 75-100 $\mathrm{mg} / \mathrm{dl}$ ), elevated lactate dehydrogenase (LDH) of 115 IU/L (normal LDH is $<50 \%$ of plasma), and increased 
protein $(3.5 \mathrm{~g} / \mathrm{dL})$. Serum LDH was $155 \mathrm{IU} / \mathrm{L}$, yielding a pleural to serum LDH ratio of 0.7, consistent with an exudative pleural effusion. Cytology was negative for malignancy on each occasion.

The patient was scheduled for video-assisted thoracic surgery (VATS) for pleural biopsy and chest tube placement for continuous drainage of the pleural effusion. On pre-operative assessment, the cardiologist recommended a right heart catheterization $(\mathrm{RCH})$ to evaluate pulmonary artery pressures. The $\mathrm{RCH}$ was significant for elevated right atrial, right ventricular, and pulmonary capillary wedge pressures. A left heart catheterization ( $\mathrm{LHC}$ ) was then planned to further evaluate the etiology of the patient's elevated right-sided pressures. Differential included left heart failure, constrictive pericarditis and restrictive cardiomyopathy. The LHC demonstrated elevated left ventricular end diastolic pressures with normal cardiac output. Hemodynamic respiratory alteration was inconclusive in distinguishing constrictive pericarditis from restrictive cardiomyopathy. An echocardiogram showed abnormal interventricular septal motion ("septal bounce"), findings consistent with both constrictive pericarditis and restrictive cardiomyopathy. The echocardiogram also showed mild pericardial thickening, making constrictive pericarditis the more likely diagnosis. Pericardiectomy, was discussed with the patient. The patient planned to be discharged and obtain a second opinion on treatment options.

Prior to planned discharge, the patient's status abruptly worsened with the development of ascites, progressive renal failure, and increasing shortness of breath requiring every other day thoracenteses. VATS at this time did not seem necessary as it would not treat the underlying condition. Because of the patient's clinical deterioration, pericardiectomy was pursued. The patient tolerated the procedure without complications. Right heart filling pressures decreased almost immediately after the procedure. The patient's renal function improved back to baseline and his right-sided pleural effusion did not re-accumulate. The patient was ambulatory on discharge. Final pathology of the pericardium showed chronic inflammation and fibrosis, consistent with constrictive pericarditis.

\section{DISCUSSION}

The majority of cases of constrictive pericarditis are idiopathic or viral in etiology, followed by post-cardiac surgery and post-mediastinal irradiation. ${ }^{1}$ Most of the patients present with symptoms of chronic heart failure. Only a minority of patients present with recurrent pleural effusion, and those who do typically have a bilateral, transudative effusion. ${ }^{2}$ That our patient had an unilateral, exudative pleural effusion was a red herring and caused significant diagnostic delay.

Postero-anterior chest $x$-ray is the initial diagnostic test of choice for evaluation of suspected pleural effusion and can identify as little as $200 \mathrm{~mL}$ of pleural fluid. Bilateral pleural effusion in a clinical setting suggestive of transudative effusion rarely require fluid analysis and can typically be treated by appropriately treating the underlying cause. Most common causes of transudative pleural effusion are increased hydrostatic pressure secondary to cardiomyopathy or liver cirrhosis. Less common causes include hypoalbuminemia, nephrotic syndrome, hypothyroidism, and mitral stenosis. The most common causes of exudative pleural effusions, on the other hand, are tuberculosis, malignancy, and parapneumonic effusion. Less common causes include pulmonary embolism, rheumatoid arthritis, pancreatitis, and post-myocardial infarction. Drugs and fungal infections are rare causes.

Our patient's abnormal right and left heart catheterization established diastolic heart failure as the cause of his pleural effusion, but the etiology of his heart failure was uncertain. Our differential included constrictive pericarditis vs. restrictive cardiomyopathy. Historically, clinically distinguishing these two entities has posed a significant challenge. Two-dimensional and Doppler echocardiography, pericardial visualization with CT scan or MRI and cardiac catheterization may be useful, but the diagnosis may remain equivocal after these tests in some patients. ${ }^{3}$ Both cause diastolic heart failure with abnormal ventricular filling pressures. Typical hemodynamic measures during cardiac catheterization include early rapid filling and equalization of end-diastolic pressures in all four cardiac chambers, but these may also be present in patients with restrictive cardiomyopathy. Some authors have suggested that assessing dynamic respiratory changes that can be observed in patients with constrictive pericarditis during 
cardiac catheterization may help distinguish these patients. ${ }^{4}$ Definitive diagnosis requires pericardial or endomyocardial biopsy.

Despite the difficulty, clinically distinguishing constrictive pericarditis and restrictive cardiomyopathy is crucial, as their treatment differs greatly. Removing the fibrotic pericardium encasing the normal myocardium, a procedure known as "pericardial stripping" or pericardiectomy, treats constrictive pericarditis. With restrictive cardiomyopathy, however, the myocardium itself is impeding normal diastolic filling, and, thus, treatment is heart transplant.

Diagnostic uncertainty was a challenge for our patient. Our patient initially had a normal echocardiogram, but as his disease worsened his echocardiogram displayed a picture consistent with constrictive physiology, including mild thickening of pericardium and abnormal interventricular septal motion ("septal bounce"). Hemodynamic studies were inconclusive and the patient's abrupt clinical decline forced us to proceed with pericardiectomy based on clinical suspicion. Prior to pericardiectomy, our patient was warned that were pericardiectomy unsuccessful, indicating either myocardial atrophy due to prolonged constriction or a true diagnosis of restrictive cardiomyopathy, he would require a heart transplant.

Complete pericardiectomy remains the treatment of choice for constrictive pericarditis, as compared to partial pericardiectomy, and has been associated with lower peri-operative mortality and improved long-term survival. Like our patient, most patients with constrictive pericarditis have significant clinical improvement following pericardiectomy. The peri-operative mortality rate of pericardiectomy is $6 \%$, most frequently secondary to low-output cardiac failure. Independent risk factors for increased risk of late mortality include increased age, higher pre-operative New York Heart Association (NYHA) class (class III and IV) and prior mediastinal irradiation. Myocardial atrophy after prolonged constriction can cause residual heart failure post-operatively despite successful pericardiectomy. ${ }^{5}$

\section{KEY POINTS}

Constrictive pericarditis is an infrequent cause of unilateral pleural effusion. This case was notable because etiology of his unilateral pleural effusion was unclear and his clinical status deteriorated quickly while the workup of his effusion was being completed. Constrictive pericarditis and restrictive cardiomyopathy must be on the differential when the etiology of pleural effusion is unclear. Imaging can suggest constrictive pericarditis; however, cardiac catheterization can further evaluate the patient's hemodynamics and strengthen the diagnosis. ${ }^{5}$ Definitive diagnosis is made with pericardial biopsy. The treatment for constrictive pericarditis is total or partial pericardiectomy.

\section{REFERENCES}

1. Bertog SC, Thambidorai SK, Parakh K, Schoenhagen P, Ozduran V, Houghtaling PL, et al. Constrictive pericarditis: Etiology and cause-specific survival after pericardiectomy. J Am Coll Cardiol. 2004 Apr 21; 43(8): 1445-52.

2. Hooper C, Lee YC, Maskell N, BTS Pleural Guideline Group. Investigation of a unilateral pleural effusion in adults: British thoracic society pleural disease guideline 2010. Thorax. 2010 Aug:65 Suppl 2: ii4-17.

3. Maisch B, Seferovic PM, Ristic AD, Erbel R, Rienmuller R, Adler $Y$, et al. Guidelines on the diagnosis and management of pericardial diseases executive summary; the task force on the diagnosis and management of pericardial diseases of the european society of cardiology. Eur Heart J. 2004 Apr; 25(7): 587-610.

4. Talreja DR, Nishimura RA, Oh JK, Holmes DR. Constrictive pericarditis in the modern era: Novel criteria for diagnosis in the cardiac catheterization laboratory. J Am Coll Cardiol. 2008 Jan 22; 51(3): 315-9.

5. Schwefer M, Aschenbach R, Heidemann J, Mey C, Lapp H. Constrictive pericarditis, still a diagnostic challenge: Comprehensive review of clinical management. Eur J Cardiothorac Surg. 2009 Sep; 36(3): 502-10. 